Article

\title{
Some Summation Theorems for Generalized Hypergeometric Functions
}

\author{
Mohammad Masjed-Jamei ${ }^{1,2, *}$ and Wolfram Koepf ${ }^{1}$
}

1 Department of Mathematics, University of Kassel, Heinrich-Plett-Str. 40, D-34132 Kassel, Germany; koepf@mathematik.uni-kassel.de

2 Department of Mathematics, K. N. Toosi University of Technology, P.O. Box 16315-1618, 11369 Tehran, Iran

* Correspondence: mmjamei@kntu.ac.ir or mmjamei@yahoo.com

Received: 2 May 2018; Accepted: 6 June 2018; Published: 8 June 2018

check for updates

\begin{abstract}
Essentially, whenever a generalized hypergeometric series can be summed in terms of gamma functions, the result will be important as only a few such summation theorems are available in the literature. In this paper, we apply two identities of generalized hypergeometric series in order to extend some classical summation theorems of hypergeometric functions such as Gauss, Kummer, Dixon, Watson, Whipple, Pfaff-Saalschütz and Dougall formulas and also obtain some new summation theorems in the sequel.
\end{abstract}

Keywords: generalized hypergeometric series; Gauss and confluent hypergeometric functions; classical summation theorems of hypergeometric functions

MSC: 33C20; 33C05; 65B10

\section{Introduction}

Let $\mathbb{R}$ and $\mathbb{C}$ denote the sets of real and complex numbers and $z$ be a complex variable. For real or complex parameters $a$ and $b$, the generalized binomial coefficient:

$$
\left(\begin{array}{l}
a \\
b
\end{array}\right)=\frac{\Gamma(a+1)}{\Gamma(b+1) \Gamma(a-b+1)}=\left(\begin{array}{c}
a \\
a-b
\end{array}\right) \quad(a, b \in \mathbb{C}),
$$

in which:

$$
\Gamma(z)=\int_{0}^{\infty} x^{z-1} e^{-x} d x,
$$

denotes the well-known gamma function for $\operatorname{Re}(z)>0$, can be reduced to the particular case:

$$
\left(\begin{array}{l}
a \\
n
\end{array}\right)=\frac{(-1)^{n}(-a)_{n}}{n !}
$$

where $(a)_{b}$ denotes the Pochhammer symbol [1] given by:

$$
(a)_{b}=\frac{\Gamma(a+b)}{\Gamma(a)}=\left\{\begin{array}{cc}
1 & (b=0, \\
a(a+1) \ldots(a+n-1) & (b \in \mathbb{C} \backslash\{0\}), a \in \mathbb{C}) .
\end{array}\right.
$$

Based on Pochhammer's symbol (1), the generalized hypergeometric functions [2]:

$$
{ }_{p} F_{q}\left(\begin{array}{cc}
a_{1}, \ldots & , a_{p} \\
b_{1}, \ldots & , b_{q}
\end{array} \mid z\right)=\sum_{k=0}^{\infty} \frac{\left(a_{1}\right)_{k} \ldots\left(a_{p}\right)_{k}}{\left(b_{1}\right)_{k} \ldots\left(b_{q}\right)_{k}} \frac{z^{k}}{k !}
$$


are indeed a Taylor series expansion for a function, say $f$, as $\sum_{k=0}^{\infty} c_{k}^{*} z^{k}$ with $c_{k}^{*}=f^{(k)}(0) / k$ !, for which the ratio of successive terms can be written as:

$$
\frac{c_{k+1}^{*}}{c_{k}^{*}}=\frac{\left(k+a_{1}\right)\left(k+a_{2}\right) \ldots\left(k+a_{p}\right)}{\left(k+b_{1}\right)\left(k+b_{2}\right) \ldots\left(k+b_{q}\right)(k+1)} .
$$

According to the ratio test [3,4], the series (2) is convergent for any $p \leq q+1$. In fact, it converges in $|z|<1$ for $p=q+1$, converges everywhere for $p<q+1$ and converges nowhere $(z \neq 0)$ for $p>q+1$. Moreover, for $p=q+1$, it absolutely converges for $|z|=1$ if the condition:

$$
A^{*}=\operatorname{Re}\left(\sum_{j=1}^{q} b_{j}-\sum_{j=1}^{q+1} a_{j}\right)>0,
$$

holds and is conditionally convergent for $|z|=1$ and $z \neq 1$ if $-1<A^{*} \leq 0$ and is finally divergent for $|z|=1$ and $z \neq 1$ if $A^{*} \leq-1$.

There are two important cases of the series (2) arising in many physics problems [5,6]. The first case (convergent in $|z| \leq 1$ ) is the Gauss hypergeometric function:

$$
y={ }_{2} F_{1}\left(\begin{array}{cc|c}
a & b & z \\
c & z
\end{array}\right)=\sum_{k=0}^{\infty} \frac{(a)_{k}(b)_{k}}{(c)_{k}} \frac{z^{k}}{k !}
$$

with the integral representation:

$$
\begin{array}{r}
{ }_{2} F_{1}\left(\begin{array}{cc|c}
a & b & z \\
c & z
\end{array}\right)=\frac{\Gamma(c)}{\Gamma(b) \Gamma(c-b)} \int_{0}^{1} t^{b-1}(1-t)^{c-b-1}(1-t z)^{-a} d t \\
\quad(\operatorname{Re} c>\operatorname{Re} b>0 ;|\arg (1-z)|<\pi) .
\end{array}
$$

Replacing $z=1$ in (3) directly leads to the well-known Gauss identity [1]:

$$
{ }_{2} F_{1}\left(\begin{array}{cc|c}
a & b & 1 \\
c & 1
\end{array}\right)=\frac{\Gamma(c) \Gamma(c-a-b)}{\Gamma(c-a) \Gamma(c-b)} \quad \operatorname{Re}(c-a-b)>0 .
$$

The second case, which converges everywhere, is the Kummer confluent hypergeometric function:

$$
y={ }_{1} F_{1}\left(\begin{array}{l|l}
b & z \\
c & z
\end{array}\right)=\sum_{k=0}^{\infty} \frac{(b)_{k}}{(c)_{k}} \frac{z^{k}}{k !},
$$

with the integral representation:

$$
{ }_{1} F_{1}\left(\begin{array}{l|l}
b & z \\
c & z
\end{array}\right)=\frac{\Gamma(c)}{\Gamma(b) \Gamma(c-b)} \int_{0}^{1} t^{b-1}(1-t)^{c-b-1} e^{z t} d t,
$$

$$
(\operatorname{Re} c>\operatorname{Re} b>0 ;|\arg (1-z)|<\pi) .
$$

Essentially, whenever a generalized hypergeometric series can be summed in terms of gamma functions, the result will be important as only a few such summation theorems are available in the literature; see, e.g., [7-13]. In this sense, the classical summation theorems such as Kummer and Gauss for ${ }_{2} F_{1}$, Dixon, Watson, Whipple and Pfaff-Saalschütz for ${ }_{3} F_{2}$, Whipple for ${ }_{4} F_{3}$, Dougall for ${ }_{5} F_{4}$ and Dougall for ${ }_{7} F_{6}$ are well known [1,14]. In this paper, we apply two identities of generalized hypergeometric functions in order to obtain some new summation theorems and extend the above-mentioned classical theorems. For this purpose, we should first recall the classical theorems as follows. 
* Kummer's theorem ([1], p. 108):

$$
{ }_{2} F_{1}\left(\begin{array}{c|c}
a, b \\
1+a-b
\end{array} \mid-1\right)=\frac{\Gamma(1+a-b) \Gamma(1+(a / 2))}{\Gamma(1-b+(a / 2)) \Gamma(1+a)} .
$$

* Second Gauss theorem ([1], p. 108):

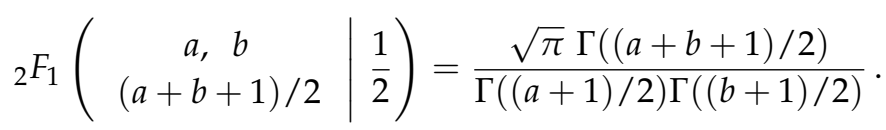

* Bailey's theorem ([1], p. 108):

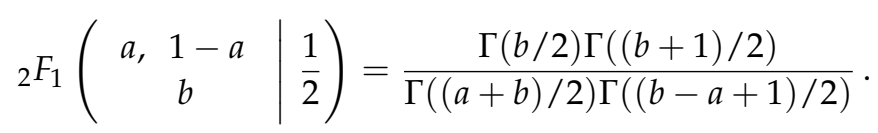

* Dixon's theorem ([1], p. 108):

$$
{ }_{3} F_{2}\left(\begin{array}{c|c}
a, b, c \\
1+a-b, 1+a-c
\end{array} \mid 1\right)=\frac{\Gamma(1+a / 2) \Gamma(1+a-b) \Gamma(1+a-c) \Gamma(1-b-c+a / 2)}{\Gamma(1+a) \Gamma(1-b+a / 2) \Gamma(1-c+a / 2) \Gamma(1+a-b-c)} .
$$

* Watson's theorem ([1], p. 108):

$$
{ }_{3} F_{2}\left(\begin{array}{c|c}
a, b, c \\
(a+b+1) / 2,2 c
\end{array} \mid 1\right)=\frac{\sqrt{\pi} \Gamma(1+c / 2) \Gamma((a+b+1) / 2) \Gamma(c-(a+b-1) / 2)}{\Gamma((a+1) / 2) \Gamma((b+1) / 2) \Gamma(c-(a-1) / 2) \Gamma(c-(b-1) / 2)} .
$$

* Whipple's theorem ([1], p. 108):

$$
\begin{aligned}
{ }_{3} F_{2}\left(\begin{array}{cc|c}
a, 1-a, & c & 1 \\
c, 2 b-c+1 & 1
\end{array}\right) \\
\quad=\frac{\pi 2^{1-2 b} \Gamma(c) \Gamma(2 b-c+1)}{\Gamma((a+c) / 2) \Gamma(b+(a-c+1) / 2) \Gamma((1-a+c) / 2) \Gamma(b+1-(a+c) / 2)} .
\end{aligned}
$$

* Pfaff-Saalschütz theorem ([1], p. 108):

$$
{ }_{3} F_{2}\left(\begin{array}{c|c}
a, b,-n \\
c, 1+a+b-c-n
\end{array} \mid 1\right)=\frac{(c-a)_{n}(c-b)_{n}}{(c)_{n}(c-a-b)_{n}} .
$$

* Second Whipple theorem ([1], p. 108):

$$
{ }_{4} F_{3}\left(\begin{array}{c|c}
a, 1+a / 2, & b, c \\
a / 2, & a-b+1, a-c+1
\end{array} \mid-1\right)=\frac{\Gamma(a-b+1) \Gamma(a-c+1)}{\Gamma(a+1) \Gamma(a-b-c+1)} .
$$

* Dougall's theorem ([1], p. 108):

$$
{ }_{5} F_{4}\left(\begin{array}{c|c}
a, 1+a / 2, c, d, e \\
a / 2, a-c+1, a-d+1, a-e+1 & 1
\end{array}\right)
$$




$$
=\frac{\Gamma(a-c+1) \Gamma(a-d+1) \Gamma(a-e+1) \Gamma(a-c-d-e+1)}{\Gamma(a+1) \Gamma(a-d-e+1) \Gamma(a-c-e+1) \Gamma(a-c-d+1)} .
$$

* Second Dougall theorem ([1], p. 108):

$$
\begin{aligned}
& { }_{7} F_{6}\left(\begin{array}{c|c}
a, 1+a / 2, b, c, d, 1+2 a-b-c+n,-n \\
a / 2, a-b+1, a-c+1, a-d+1, b+c+d-a-n, a+1+n & 1
\end{array}\right) \\
& =\frac{(a+1)_{n}(a-b-c+1)_{n}(a-b-d+1)_{n}(a-c-d+1)_{n}}{(a+1-b)_{n}(a+1-c)_{n}(a+1-d)_{n}(a+1-b-c-d)_{n}} .
\end{aligned}
$$

In order to derive the first identity and only for simplicity, we will use the following symbol for representing finite sums of hypergeometric series:

$$
\stackrel{(m)}{p} q\left(\begin{array}{ccc}
a_{1}, \ldots & , a_{p} \\
b_{1}, & \ldots & , b_{q}
\end{array} \mid z\right)=\sum_{k=0}^{m} \frac{\left(a_{1}\right)_{k} \ldots\left(a_{p}\right)_{k}}{\left(b_{1}\right)_{k} \ldots\left(b_{q}\right)_{k}} \frac{z^{k}}{k !} .
$$

For instance, we have:

$$
{ }_{p} \stackrel{(-1)}{F}_{q}(z)=0, \stackrel{(0)}{F}_{q}(z)=1 \text { and } \stackrel{(1)}{F}_{q}(z)=1+\frac{a_{1} \ldots a_{p}}{b_{1} \ldots b_{q}} z .
$$

\section{First Hypergeometric Identity}

Let $m, n$ be two natural numbers so that $n \leq m$. By referring to Relation (1), since:

$$
\frac{(n)_{k}}{(m)_{k}}=\frac{\Gamma(k+n) \Gamma(m)}{\Gamma(k+m) \Gamma(n)}=\frac{\Gamma(m)}{\Gamma(n)} \frac{1}{(k+n)(k+n+1) \ldots(k+m-1)},
$$

substituting (15) in a special case of (2) yields:

$$
\begin{aligned}
& { }_{p} F_{q}\left(\begin{array}{cc}
a_{1}, \ldots, a_{p-1}, & n \\
b_{1}, \ldots, b_{q-1}, & m
\end{array} \mid z\right)=\frac{\Gamma(m)}{\Gamma(n)} \sum_{k=0}^{\infty} \frac{\left(a_{1}\right)_{k} \ldots\left(a_{p-1}\right)_{k}}{\left(b_{1}\right)_{k} \ldots\left(b_{q-1}\right)_{k}} \frac{(k+1)(k+2) \ldots(k+n-1) z^{k}}{(k+m-1) !} \\
& =\frac{\Gamma(m)}{\Gamma(n)} \sum_{j=m-1}^{\infty} \frac{\left(a_{1}\right)_{j-m+1} \ldots\left(a_{p-1}\right)_{j-m+1}}{\left(b_{1}\right)_{j-m+1} \ldots\left(b_{q-1}\right)_{j-m+1}} \frac{(j+2-m)(j+3-m) \ldots(j-m+n) z^{j-m+1}}{j !} .
\end{aligned}
$$

Relation (16) shows that we encounter a complicated computational problem that cannot be easily evaluated. However, some particular cases such as $n=1$ and $n=2$ can be directly computed. We leave other cases as open problems.

The case $n=1$ leads to a known result in the literature [14], because: 


$$
\begin{aligned}
& { }_{p} F_{q}\left(\begin{array}{cc|c}
a_{1}, \ldots, a_{p-1}, & 1 \\
b_{1}, \ldots, b_{q-1}, & m & z
\end{array}\right)=\Gamma(m) \sum_{j=m-1}^{\infty} \frac{\left(a_{1}\right)_{j-m+1} \ldots\left(a_{p-1}\right)_{j-m+1}}{\left(b_{1}\right)_{j-m+1} \cdots\left(b_{q-1}\right)_{j-m+1}} \frac{z^{j-m+1}}{j !} \\
& =\Gamma(m)\left(\sum_{j=0}^{\infty} \frac{\left(a_{1}\right)_{j-m+1} \cdots\left(a_{p-1}\right)_{j-m+1}}{\left(b_{1}\right)_{j-m+1} \cdots\left(b_{q-1}\right)_{j-m+1}} \frac{z^{j-m+1}}{j !}-\sum_{j=0}^{m-2} \frac{\left(a_{1}\right)_{j-m+1} \cdots\left(a_{p-1}\right)_{j-m+1}}{\left(b_{1}\right)_{j-m+1} \cdots\left(b_{q-1}\right)_{j-m+1}} \frac{z^{j-m+1}}{j !}\right),
\end{aligned}
$$

and since:

$$
(a)_{j-m+1}=\frac{\Gamma(a-m+1)}{\Gamma(a)}(a-m+1)_{j},
$$

Relation (17) is simplified as:

$$
\begin{aligned}
& { }_{p} F_{q}\left(\begin{array}{cc|c}
a_{1}, \ldots, a_{p-1}, & 1 \\
b_{1}, \ldots, b_{q-1}, & m & z
\end{array}\right) \\
& =\frac{\Gamma\left(b_{1}\right) \ldots \Gamma\left(b_{q-1}\right)}{\Gamma\left(a_{1}\right) \ldots \Gamma\left(a_{p-1}\right)} \frac{\Gamma\left(a_{1}-m+1\right) \ldots \Gamma\left(a_{p-1}-m+1\right)}{\Gamma\left(b_{1}-m+1\right) \ldots \Gamma\left(b_{q-1}-m+1\right)} \frac{(m-1) !}{z^{m-1}} \\
& \times\left({ }_{p-1} F_{q-1}\left(\begin{array}{c}
a_{1}-m+1, \ldots, \\
b_{1}-m+1, \ldots, b_{q-1}-m+1 \\
b_{q-1}-m+1
\end{array} \mid z\right)\right.
\end{aligned}
$$

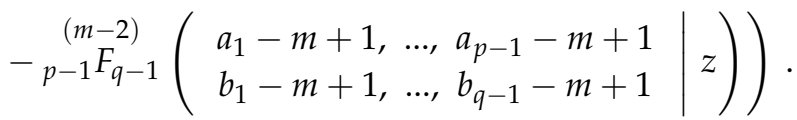

However, the interesting point is that using Relation (18), we can obtain various special cases that extend all classical summation theorems as follows.

Special Case 1. When $p=3, q=2$ and $x=1$, Relation (18) is simplified as:

$$
\begin{aligned}
& { }_{3} F_{2}\left(\begin{array}{cc|c}
a, & b, & 1 \\
c, & 1
\end{array}\right)=\frac{\Gamma(m) \Gamma(c) \Gamma(a-m+1) \Gamma(b-m+1)}{\Gamma(a) \Gamma(b) \Gamma(c-m+1)} \\
& \times\left(\frac{\Gamma(c-m+1) \Gamma(c-a-b+m-1)}{\Gamma(c-a) \Gamma(c-b)}-{ }_{2}^{(m-2)} F_{1}\left(\begin{array}{c|c}
a-m+1, b-m+1 & 1 \\
c-m+1 & 1
\end{array}\right)\right) .
\end{aligned}
$$

For $m=1$, Relation (19) exactly gives Formula (4), while for $m=2,3$, we have:

$$
{ }_{3} F_{2}\left(\begin{array}{cc|c}
a & b, & 1 \\
c, 2 & 1
\end{array}\right)=\frac{c-1}{(a-1)(b-1)}\left(\frac{\Gamma(c-1) \Gamma(c-a-b+1)}{\Gamma(c-a) \Gamma(c-b)}-1\right)
$$

and:

$$
\begin{aligned}
{ }_{3} F_{2}\left(\begin{array}{ccc|c}
a, b & 1 & 1 \\
c, 3 & 1
\end{array}\right)=\frac{2(c-2)_{2}}{(a-2)_{2}(b-2)_{2}} & \\
& \times\left(\frac{\Gamma(c-2) \Gamma(c-a-b+2)}{\Gamma(c-a) \Gamma(c-b)}-\frac{a b+c-2 a-2 b+2}{c-2}\right) .
\end{aligned}
$$

These two formulas are given in [14].

Special Case 2. When $p=3, q=2$ and $x=-1$, by noting the Kummer theorem (5), Relation (18) is simplified as: 


$$
\begin{aligned}
& { }_{3} F_{2}\left(\begin{array}{c}
a, b, 1 \\
a-b+m, m
\end{array} \mid-1\right)=(-1)^{m-1} \frac{\Gamma(m) \Gamma(a-b+m) \Gamma(a-m+1) \Gamma(b-m+1)}{\Gamma(a) \Gamma(b) \Gamma(a-b+1)} \\
& \quad \times\left(\frac{\Gamma(a-b+1) \Gamma(1+(a-m+1) / 2)}{\Gamma(2+a-m) \Gamma(m-b+(a-m+1) / 2)}-{ }_{2}{ }_{2} F_{1}\left(\begin{array}{c}
a-m+1, b-m+1 \\
a-b+1
\end{array} \mid-1\right)\right) .
\end{aligned}
$$

For $m=1$, Relation (20) exactly gives the Kummer formula, while for $m=2,3$, we have:

$$
{ }_{3} F_{2}\left(\begin{array}{c|c}
a, b, 1 \\
a-b+2,2
\end{array} \mid-1\right)=\frac{a-b+1}{(a-1)(b-1)}\left(1-\frac{\Gamma(a-b+1) \Gamma(1+(a-1) / 2)}{\Gamma(a) \Gamma(-b+2+(a-1) / 2)}\right),
$$

and:

$$
{ }_{3} F_{2}\left(\begin{array}{c}
a, b, 1 \\
a-b+3,3
\end{array} \mid-1\right)=\frac{2(a-b+1)_{2}}{(a-2)_{2}(b-2)_{2}}\left(\frac{\Gamma(a-b+1) \Gamma(a / 2)}{\Gamma(a-1) \Gamma(-b+2+a / 2)}-\frac{3 a+b-a b-3}{a-b+1}\right) .
$$

Special Case 3. When $p=3, q=2$ and $x=1 / 2$, by noting the second kind of Gauss Formula (6), Relation (18) is simplified as:

$$
\begin{aligned}
& { }_{3} F_{2}\left(\begin{array}{c|c}
a, b, 1 & 1 \\
(a+b+1) / 2, & m
\end{array}\right)=(2)^{m-1} \\
& \times \frac{\Gamma(m) \Gamma((a+b+1) / 2) \Gamma(a-m+1) \Gamma(b-m+1)}{\Gamma(a) \Gamma(b) \Gamma(-m+1+(a+b+1) / 2)} \\
& \times\left(\frac{\sqrt{\pi} \Gamma(-m+1+(a+b+1) / 2)}{\Gamma(1+(a-m) / 2) \Gamma(1+(b-m) / 2)}-{ }_{2}^{2} F_{1}\left(\begin{array}{cc|c}
a-m+1, b-m+1 & 1 \\
-m+1+(a+b+1) / 2 & \frac{(m-2)}{2}
\end{array}\right) .\right.
\end{aligned}
$$

For $m=1$, Relation (21) exactly gives the second kind of Gauss formula, while for $m=2,3$, we have:

$$
{ }_{3} F_{2}\left(\begin{array}{c|c}
a, b, 1 & 1 \\
(a+b+1) / 2,2 & \frac{2}{2}
\end{array}\right)=\frac{a+b-1}{(a-1)(b-1)}\left(\frac{\sqrt{\pi} \Gamma(-1+(a+b+1) / 2)}{\Gamma(a / 2) \Gamma(b / 2)}-1\right),
$$

and:

$$
\begin{aligned}
& { }_{3} F_{2}\left(\begin{array}{c|c}
a, b, 1 & 1 \\
(a+b+1) / 2,3 & \frac{1}{2}
\end{array}\right)=\frac{2(a+b-1)(a+b-3)}{(a-2)_{2}(b-2)_{2}} \\
& \times\left(\frac{\sqrt{\pi} \Gamma((a+b-3) / 2)}{\Gamma((a-1) / 2) \Gamma((b-1) / 2)}-\frac{a b-a-b+1}{a+b-3}\right) .
\end{aligned}
$$

Special Case 4. When $p=3, q=2$ and $x=1 / 2$, by noting the Bailey theorem (7), Relation (18) is simplified as: 


$$
\begin{aligned}
& { }_{3} F_{2}\left(\begin{array}{cc|c}
a, & 2 m-a-1,1 & \frac{1}{2} \\
b, & m
\end{array}\right)=(2)^{m-1} \frac{\Gamma(m) \Gamma(b) \Gamma(a-m+1) \Gamma(m-a)}{\Gamma(a) \Gamma(2 m-a-1) \Gamma(b-m+1)} \\
& \quad \times\left(\frac{\Gamma((b-m+1) / 2) \Gamma((b-m+2) / 2)}{\Gamma(-m+1+(a+b) / 2) \Gamma((b-a+1) / 2)}-{ }_{2}^{(m-2)} F_{1}\left(\begin{array}{cc|c}
a-m+1, m-a & 1 \\
b-m+1 & \frac{(}{2}
\end{array}\right)\right) .
\end{aligned}
$$

For $m=1$, Relation (22) exactly gives the Bailey formula, while for $m=2,3$, we have:

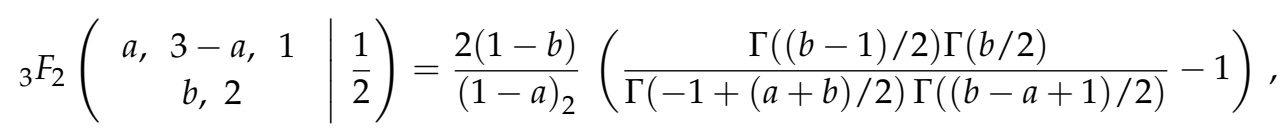

and:

$$
\begin{aligned}
& { }_{3} F_{2}\left(\begin{array}{cc|c}
a, 5-a, & 1 & 1 \\
b, 3 & \frac{8}{2}
\end{array}\right)=\frac{8(b-2)_{2}}{(a-4)_{4}} \\
& \times\left(\frac{\Gamma((b-1) / 2) \Gamma((b-2) / 2)}{\Gamma(-2+(a+b) / 2) \Gamma((b-a+1) / 2)}-\frac{5 a-a^{2}+2 b-10}{2(b-2)}\right) .
\end{aligned}
$$

Special Case 5. When $p=4, q=3$ and $x=1$, by noting the Dixon theorem (8), Relation (18) is simplified as:

$$
\begin{gathered}
{ }_{4} F_{3}\left(\begin{array}{c}
a, b, c, 1 \\
a-b+m, a-c+m, m
\end{array}\right) \\
=\frac{\Gamma(m) \Gamma(a-b+m) \Gamma(a-c+m) \Gamma(a+1-m) \Gamma(b+1-m) \Gamma(c+1-m)}{\Gamma(a) \Gamma(b) \Gamma(c) \Gamma(a-b+1) \Gamma(a-c+1)} \\
\times\left(\begin{array}{c}
\frac{\Gamma((a+3-m) / 2) \Gamma(a-b+1) \Gamma(a-c+1) \Gamma(-b-c+(a+3 m-1) / 2)}{\Gamma(a+2-m) \Gamma(-b+(a+m+1) / 2) \Gamma(-c+(a+m+1) / 2) \Gamma(a-b-c+m)} \\
(m-2) \\
-{ }_{3} F_{2}\left(\begin{array}{c}
a-m+1, b-m+1, c-m+1 \\
a-b+1, a-c+1
\end{array}\right.
\end{array}\right) .
\end{gathered}
$$

For $m=1$, Relation (23) exactly gives the Dixon formula, while for $m=2,3$, we have:

$$
\begin{array}{r}
{ }_{4} F_{3}\left(\begin{array}{c}
a, b, c, 1 \\
a-b+2, a-c+2,2
\end{array}\right)=\frac{(a-b+1)(a-c+1)}{(a-1)(b-1)(c-1)} \\
\times\left(\frac{\Gamma((a+1) / 2) \Gamma(a-b+1) \Gamma(a-c+1) \Gamma(-b-c+(a+5) / 2)}{\Gamma(a) \Gamma(-b+(a+3) / 2) \Gamma(-c+(a+3) / 2) \Gamma(a-b-c+2)}-1\right),
\end{array}
$$

and: 


$$
\begin{aligned}
& { }_{4} F_{3}\left(\begin{array}{c}
a, b, c, 1 \\
a-b+3, a-c+3,3
\end{array} \mid 1\right)=\frac{2(a-b+1)_{2}(a-c+1)_{2}}{(a-2)_{2}(b-2)_{2}(c-2)_{2}} \\
& \quad \times\left(\frac{\Gamma(a / 2) \Gamma(a-b+1) \Gamma(a-c+1) \Gamma((a / 2)-b-c+4)}{\Gamma(a-1) \Gamma((a / 2)-b+2) \Gamma((a / 2)-c+2) \Gamma(a-b-c+3)}-\frac{(a-2)(b-2)(c-2)}{(a-b+1)(a-c+1)}-1\right) .
\end{aligned}
$$

Special Case 6. When $p=4, q=3$ and $x=1$, by noting the Watson theorem (9), Relation (18) is simplified as:

$$
\begin{aligned}
& { }_{4} F_{3}\left(\begin{array}{cl|l}
a, b, & c, 1 \\
(a+b+1) / 2, & 2 c+1-m, m & 1
\end{array}\right) \\
& =\frac{\Gamma(m) \Gamma((a+b+1) / 2) \Gamma(2 c+1-m) \Gamma(a+1-m) \Gamma(b+1-m) \Gamma(c+1-m)}{\Gamma(a) \Gamma(b) \Gamma(c) \Gamma(-m+(a+b+3) / 2) \Gamma(2 c-2 m+2)}
\end{aligned}
$$

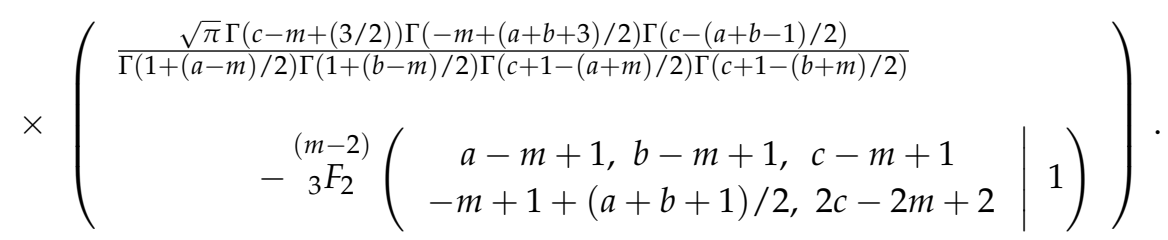

For $m=1$, Relation (24) exactly gives the Watson formula, while for $m=2,3$, we have:

$$
\begin{aligned}
{ }_{4} F_{3}\left(\begin{array}{c|c}
a, b, c, 1 \\
(a+b+1) / 2,2 c-1,2 & 1
\end{array}\right)=\frac{a+b-1}{(a-1)(b-1)} \\
\times\left(\frac{\sqrt{\pi} \Gamma(c-(1 / 2)) \Gamma((a+b-1) / 2) \Gamma(c-(a+b-1) / 2)}{\Gamma(a / 2) \Gamma(b / 2) \Gamma(c-(a / 2)) \Gamma(c-(b / 2))}-1\right),
\end{aligned}
$$

and:

$$
\begin{aligned}
& { }_{4} F_{3}\left(\begin{array}{c}
a, b, c, 1 \\
(a+b+1) / 2,2 c-2,3
\end{array} \mid 1\right)=\frac{(2 c-3)(a+b-1)(a+b-3)}{(a-2)_{2}(b-2)_{2}(c-1)} \\
& \quad \times\left(\frac{\sqrt{\pi} \Gamma(c-(3 / 2)) \Gamma((a+b-3) / 2) \Gamma(c-(a+b-1) / 2)}{\Gamma((a-1) / 2) \Gamma((b-1) / 2) \Gamma(c-(a+1) / 2) \Gamma(c-(b+1) / 2)}-\frac{(a-2)(b-2)}{a+b-3}-1\right) .
\end{aligned}
$$

Special Case 7. When $p=4, q=3$ and $x=1$, by noting the Whipple theorem (10), Relation (18) is simplified as: 


$$
\begin{aligned}
{ }_{4} F_{3}\left(\begin{array}{rll}
a, 2 m-1-a, & b & 1 \\
c, 2 b-c+1, & m & 1
\end{array}\right) \\
=\frac{\Gamma(m) \Gamma(c) \Gamma(2 b-c+1) \Gamma(a+1-m) \Gamma(m-a) \Gamma(b+1-m)}{\Gamma(a) \Gamma(2 m-1-a) \Gamma(b) \Gamma(c+1-m) \Gamma(2 b-c-m+2)} \\
\times\left(\begin{array}{r}
\frac{\pi 2^{2 m-2 b-1} \Gamma(c-m+1) \Gamma(2 b-c+2-m)}{\Gamma(-m+1+(a+c) / 2) \Gamma(-m+1+b+(a-c+1) / 2) \Gamma((1-a+c) / 2) \Gamma(b+1-(a+c) / 2)} \\
(m-2) \\
-{ }_{3} F_{2}\left(\begin{array}{c}
a-m+1, m-a, b-m+1 \\
c-m+1,2 b-c-m+2
\end{array}\right.
\end{array}\right)
\end{aligned}
$$

For $m=1$, Relation (25) exactly gives the Whipple formula, while for $m=2,3$, we have:

$$
\begin{aligned}
& { }_{4} F_{3}\left(\begin{array}{c|c}
a, 3-a, b, 1 & 1 \\
c, 2 b-c+1,2 & 1
\end{array}\right)=\frac{(c-1)(c-2 b)}{(a-2)_{2}(b-1)} \times \\
& \left(\frac{\pi 2^{3-2 b} \Gamma(c-1) \Gamma(2 b-c)}{\Gamma(-1+(a+c) / 2) \Gamma(b+(a-c-1) / 2) \Gamma((1-a+c) / 2) \Gamma(b+1-(a+c) / 2)}-1\right),
\end{aligned}
$$

and:

$$
\begin{aligned}
& { }_{4} F_{3}\left(\begin{array}{c|c}
a, 5-a, b, 1 & 1 \\
c, 2 b-c+1,3 & 1
\end{array}\right)=\frac{2(c-2)_{2}(2 b-c-1)_{2}}{(a-4)_{4}(b-2)_{2}} \\
& \times\left(\frac{\pi 2^{5-2 b} \Gamma(c-2) \Gamma(2 b-c-1)}{\Gamma(-2+(a+c) / 2) \Gamma(b+(a-c-3) / 2) \Gamma((1-a+c) / 2) \Gamma(b+1-(a+c) / 2)}\right. \\
& \left.-\frac{(a-2)(3-a)(b-2)}{(c-2)(2 b-c-1)}-1\right) \text {. }
\end{aligned}
$$

Special Case 8. When $p=4, q=3$ and $x=1$, by noting the Pfaff-Saalschütz theorem (11), Relation (18) is simplified as:

$$
\begin{aligned}
& { }_{4} F_{3}\left(\begin{array}{c}
a, b,-n+m-1,1 \\
c, 1+a+b-c-n, m
\end{array} \mid 1\right)=\frac{(m-1) !(1-c)_{m-1}(c-a-b+n)_{m-1}}{(1-a)_{m-1}(1-b)_{m-1}(n+2-m)_{m-1}} \\
& \times\left(\frac{(c-a)_{n}(c-b)_{n}}{(c+1-m)_{n}(c-a-b+m-1)_{n}}-{ }_{3}^{(m-2)}\left(\begin{array}{c}
a-m+1, b-m+1,-n \\
c-m+1,2+a+b-c-m-n
\end{array}\right)\right) .
\end{aligned}
$$

For $m=1$, Relation (26) exactly gives the Pfaff-Saalschütz formula, while for $m=2,3$, we have:

$$
\begin{aligned}
{ }_{4} F_{3}\left(\begin{array}{c|c}
a, b,-n+1,1 \\
c, 1+a+b-c-n, 2
\end{array} \mid 1\right)=\frac{(1-c)(c-a-b+n)}{n(1-a)(1-b)} & \\
& \times\left(\frac{(c-a)_{n}(c-b)_{n}}{(c-1)_{n}(c-a-b+1)_{n}}-1\right),
\end{aligned}
$$

and: 


$$
\begin{aligned}
{ }_{4} F_{3}\left(\begin{array}{c|c}
a, b,-n+2,1 \\
c, 1+a+b-c-n, 3
\end{array}\right. & 1)=\frac{2(1-c)_{2}(c-a-b+n)_{2}}{n(1-a)_{2}(1-b)_{2}(n-1)_{2}} \\
\times & \left(\frac{(c-a)_{n}(c-b)_{n}}{(c-2)_{n}(c-a-b+2)_{n}}+\frac{n(a-2)(b-2)}{(c-2)(a+b-c-n-1)}-1\right) .
\end{aligned}
$$

Special Case 9. When $p=5, q=4$ and $x=-1$, by noting the second theorem of Whipple (12), Relation (18) is simplified as:

$$
\begin{aligned}
& { }_{5} F_{4}\left(\begin{array}{c}
a,(a+m+1) / 2, b, c, 1 \\
(a+m-1) / 2, a-b+m, a-c+m, m
\end{array} \mid-1\right)=(-1)^{m-1} \times \\
& \quad \frac{\Gamma(m) \Gamma((a+m-1) / 2) \Gamma(a-b+m) \Gamma(a-c+m) \Gamma(a-m+1) \Gamma((a-m+3) / 2) \Gamma(b-m+1) \Gamma(c-m+1)}{\Gamma(a) \Gamma((a+m+1) / 2) \Gamma(b) \Gamma(c) \Gamma((a-m+1) / 2) \Gamma(a-b+1) \Gamma(a-c+1)} \\
& \quad \times\left(\frac{\Gamma(1+a-b) \Gamma(1+a-c)}{\Gamma(2-m+a) \Gamma(m+a-b-c)}-{ }_{4}^{(m-2)} F_{3}\left(\begin{array}{c}
a-m+1,(a-m+3) / 2, b-m+1, c-m+1 \\
(a-m+1) / 2, a-b+1, a-c+1
\end{array} \mid-1\right)\right) .
\end{aligned}
$$

For $m=1$, Relation (27) exactly gives the Whipple formula, while for $m=2,3$, we have:

$$
\begin{aligned}
& { }_{5} F_{4}\left(\begin{array}{c|c}
a,(a+3) / 2, b, c, 1 & -1 \\
(a+1) / 2, a-b+2, a-c+2,2 & -1
\end{array}\right) \\
& =\frac{4(a-b+1)(a-c+1)}{\left(a^{2}-1\right)(a-1)(b-1)(c-1)}\left(1-\frac{\Gamma(1+a-b) \Gamma(1+a-c)}{\Gamma(a) \Gamma(2+a-b-c)}\right),
\end{aligned}
$$

and:

$$
\begin{array}{r}
{ }_{5} F_{4}\left(\begin{array}{c}
a,(a+4) / 2, b, c, 1 \\
(a+2) / 2, a-b+3, a-c+3,3
\end{array} \mid-1\right)=\frac{2(a-b+1)_{2}(a-c+1)_{2}}{(a+2)(a-1)(b-2)_{2}(c-2)_{2}} \\
\times\left(\frac{\Gamma(1+a-b) \Gamma(1+a-c)}{\Gamma(a-1) \Gamma(3+a-b-c)}+\frac{a(b-2)(c-2)}{(a-b+1)(a-c+1)}-1\right) .
\end{array}
$$

Special Case 10. When $p=6, q=5$ and $x=1$, by noting the Dougall theorem (13), Relation (18) is simplified as:

$$
\begin{gathered}
{ }_{6} F_{5}\left(\begin{array}{c}
a,(a+m+1) / 2, c, d, e, 1 \\
(a+m-1) / 2, a-c+m, a-d+m, a-e+m, m
\end{array}\right) \\
=\Gamma(m) \Gamma((a+m-1) / 2) \Gamma(a-c+m) \\
\times \frac{\Gamma(a-d+m) \Gamma(a-e+m) \Gamma(a-m+1) \Gamma((a-m+3) / 2) \Gamma(c-m+1) \Gamma(d-m+1) \Gamma(e-m+1)}{\Gamma(a) \Gamma((a+m+1) / 2) \Gamma(c) \Gamma(d) \Gamma(e) \Gamma((a-m+1) / 2) \Gamma(a-c+1) \Gamma(a-d+1) \Gamma(a-e+1)} \\
\times\left(\begin{array}{c}
\frac{\Gamma(a-c+1) \Gamma(a-d+1) \Gamma(a-e+1) \Gamma(a-c-d-e+2 m-1)}{\Gamma(a+2-m) \Gamma(a-d-e+m) \Gamma(a-c-e+m) \Gamma(a-c-d+m)} \\
-(m-2) \\
-{ }_{5} F_{4}\left(\begin{array}{c}
a-m+1,(a-m+3) / 2, c-m+1, d-m+1, e-m+1 \\
(a-m+1) / 2, a-c+1, a-d+1, a-e+1
\end{array}\right.
\end{array}\right) .
\end{gathered}
$$


For $m=1$, Relation (28) exactly gives the Dougall formula, while for $m=2,3$, we have:

$$
\begin{array}{r}
{ }_{6} F_{5}\left(\begin{array}{r}
a,(a+3) / 2, \\
(a+1) / 2, a-c, e, 1
\end{array}\right) \\
=\frac{(a-c+1)(a-d+1)(a-e+1)}{(c-1)(d-1)(e-1)(a+1)} \\
\times\left(\frac{\Gamma(a-c+1) \Gamma(a-d+1) \Gamma(a-e+1) \Gamma(a-c-d-e+3)}{\Gamma(a) \Gamma(a-d-e+2) \Gamma(a-c-e+2) \Gamma(a-c-d+2)}-1\right),
\end{array}
$$

and:

$$
\begin{gathered}
{ }_{6} F_{5}\left(\begin{array}{c|c}
a, & (a+4) / 2, c, d, e, 1 \\
(a+2) / 2, a-c+3, & a-d+3, a-e+3,3
\end{array}\right) \\
=\frac{2(a-c+1)_{2}(a-d+1)_{2}(a-e+1)_{2}}{(a-1)(a+2)(c-2)_{2}(d-2)_{2}(e-2)_{2}} \\
\times\left(\frac{\Gamma(a-c+1) \Gamma(a-d+1) \Gamma(a-e+1) \Gamma(a-c-d-e+5)}{\Gamma(a-1) \Gamma(a-d-e+3) \Gamma(a-c-e+3) \Gamma(a-c-d+3)}\right. \\
\left.-\frac{a(c-2)(d-2)(e-2)}{(a-c+1)(a-d+1)(a-e+1)}-1\right) .
\end{gathered}
$$

Special Case 11. When $p=8, q=7$ and $x=1$, by noting the second theorem of Dougall (14), Relation (18) is simplified as:

$$
\begin{gathered}
{ }_{8} F_{7}\left(\begin{array}{c}
a,(a+m+1) / 2, b, c, d, 2 a-b-c-d+2 m-1+n, m-n-1,1 \\
(a+m-1) / 2, a-b+m, a-c+m, a-d+m, b+c+d-a+1-m-n, a+n+1, m
\end{array} \mid 1\right) \\
=(-1)^{m-1}(m-1) ! \times \\
\frac{((3-a-m) / 2)_{m-1}(1-a+b-m)_{m-1}(1-a+c-m)_{m-1}(1-a+d-m)_{m-1}(m+n+a-b-c-d)_{m-1}(-a-n)_{m-1}}{((1-a-m) / 2)_{m-1}(1-a)_{m-1}(1-b)_{m-1}(1-c)_{m-1}(1-d)_{m-1}(b+c+d-2 a+2-2 m-n)_{m-1}(n+2-m)_{m-1}} \\
\times\left(\begin{array}{c}
\frac{(a-m+2)_{n}(a-b-c+m)_{n}(a-b-d+m)_{n}(a-c-d+m)_{n}}{(a-b+1)_{n}(a-c+1)_{n}(a-d+1)_{n}(a-b-c-d+2 m-1)_{n}} \\
-{ }^{(m-2)}\left(\begin{array}{c}
a-m+1,(a-m+3) / 2, b-m+1, c-m+1, d-m+1,2 a-b-c-d+m+n,-n \\
(a-m+1) / 2, a-b+1, a-c+1, a-d+1, b+c+d-a+2-2 m-n, a-m+n+2
\end{array}\right.
\end{array}\right)
\end{gathered}
$$

For $m=1$, Relation (29) exactly gives the Dougall formula, while for $m=2,3$, we have: 


$$
\begin{gathered}
{ }_{8} F_{7}\left(\begin{array}{c}
a,(a+3) / 2, b, c, d, 2 a-b-c-d+3+n,-n+1,1 \\
(a+1) / 2, a-b+2, a-c+2, a-d+2, b+c+d-a-1-n, a+n+1,2
\end{array} \mid 1\right) \\
=\frac{(-a+b-1)(-a+c-1)(-a+d-1)(n+2+a-b-c-d)(a+n)}{n(1+a)(1-b)(1-c)(1-d)(b+c+d-2 a-2-n)} \\
\times\left(1-\frac{(a)_{n}(a-b-c+2)_{n}(a-b-d+2)_{n}(a-c-d+2)_{n}}{(a-b+1)_{n}(a-c+1)_{n}(a-d+1)_{n}(a-b-c-d+3)_{n}}\right),
\end{gathered}
$$

and:

$$
\begin{gathered}
{ }_{8} F_{7}\left(\begin{array}{c}
a,(a+4) / 2, b, c, d, 2 a-b-c-d+5+n,-n+2,1 \\
(a+2) / 2, a-b+3, a-c+3, a-d+3, b+c+d-a-2-n, a+n+1,3
\end{array} \mid 1\right) \\
=\frac{(a-2)(-a+b-2)_{2}(-a+c-2)_{2}(-a+d-2)_{2}(3+n+a-b-c-d)_{2}(-a-n)_{2}}{(a+2)(1-a)_{2}(1-b)_{2}(1-c)_{2}(1-d)_{2}(b+c+d-2 a-4-n)_{2}(n-1)_{2}} \\
\times\left(\begin{array}{c}
\frac{(a-1)_{n}(a-b-c+3)_{n}(a-b-d+3)_{n}(a-c-d+3)_{n}}{(a-b+1)_{n}(a-c+1)_{n}(a-d+1)_{n}(a-b-c-d+5)_{n}} \\
+\frac{n a(b-2)(c-2)(d-2)(2 a-b-c-d+3+n)}{(a-b+1)(a-c+1)(a-d+1)(b+c+d-a-n-4)(n+a-1)}-1
\end{array}\right) .
\end{gathered}
$$

Remark 1. There are two further special cases, which however do not belong to classical summation theorems. When $p=q=1$, Relation (18) is simplified as:

$$
{ }_{1} F_{1}\left(\begin{array}{c|c}
1 & z \\
m & z
\end{array}\right)=\frac{(m-1) !}{z^{m-1}}\left(e^{z}-\sum_{j=0}^{m-2} \frac{z^{j}}{j !}\right),
$$

and when $p=q+1=2$, it yields:

$$
{ }_{2} F_{1}\left(\begin{array}{c|c}
a, & 1 \\
m & z
\end{array}\right)=\frac{(m-1) !}{z^{m-1}} \frac{\Gamma(a-m+1)}{\Gamma(a)}\left((1-z)^{m-a-1}-\sum_{j=0}^{m-2}(a-m+1)_{j} \frac{z^{j}}{j !}\right) .
$$

Similarly, for the case $n=2$, Relation (16) changes to:

$$
\begin{aligned}
& { }_{p} F_{q}\left(\begin{array}{ccc}
a_{1}, \ldots & a_{p-1}, & 2 \\
b_{1}, \ldots & b_{q-1}, & m
\end{array} \mid z\right)=\frac{\Gamma(m)}{z^{m-1}} \sum_{j=m-1}^{\infty} \frac{\left(a_{1}\right)_{j-m+1} \ldots\left(a_{p-1}\right)_{j-m+1}}{\left(b_{1}\right)_{j-m+1} \ldots\left(b_{q-1}\right)_{j-m+1}} \frac{(j+2-m) z^{j}}{j !} \\
& =\frac{\Gamma(m)}{z^{m-1}}\left(z \sum_{r=m-2}^{\infty} \frac{\left(a_{1}\right)_{j-m+2} \ldots\left(a_{p-1}\right)_{j-m+2}}{\left(b_{1}\right)_{j-m+2} \ldots\left(b_{q-1}\right)_{j-m+2}} \frac{z^{r}}{r !}+(2-m) \sum_{j=m-1}^{\infty} \frac{\left(a_{1}\right)_{j-m+1} \ldots\left(a_{p-1}\right)_{j-m+1}}{\left(b_{1}\right)_{j-m+1} \ldots\left(b_{q-1}\right)_{j-m+1}} \frac{z^{j}}{j !}\right) \\
& =\frac{(m-1) !}{z^{m-2}} \frac{\Gamma\left(b_{1}\right) \ldots \Gamma\left(b_{q-1}\right)}{\Gamma\left(a_{1}\right) \ldots \Gamma\left(a_{p-1}\right)} \frac{\Gamma\left(a_{1}-m+2\right) \ldots \Gamma\left(a_{p-1}-m+2\right)}{\Gamma\left(b_{1}-m+2\right) \ldots \Gamma\left(b_{q-1}-m+2\right)}
\end{aligned}
$$




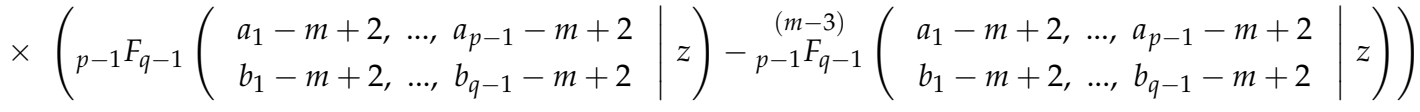

$$
\begin{aligned}
& +(2-m) \frac{(m-1) !}{z^{m-1}} \frac{\Gamma\left(b_{1}\right) \ldots \Gamma\left(b_{q-1}\right)}{\Gamma\left(a_{1}\right) \ldots \Gamma\left(a_{p-1}\right)} \frac{\Gamma\left(a_{1}-m+1\right) \ldots \Gamma\left(a_{p-1}-m+1\right)}{\Gamma\left(b_{1}-m+1\right) \ldots \Gamma\left(b_{q-1}-m+1\right)}
\end{aligned}
$$

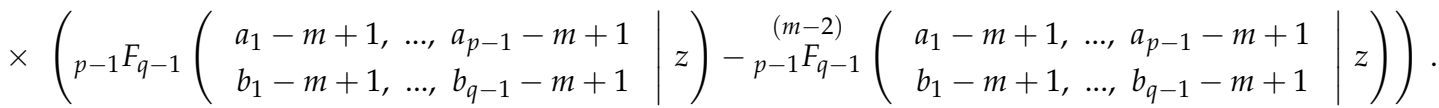

Therefore:

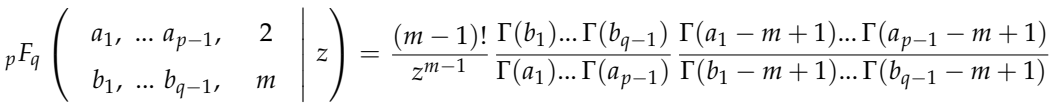

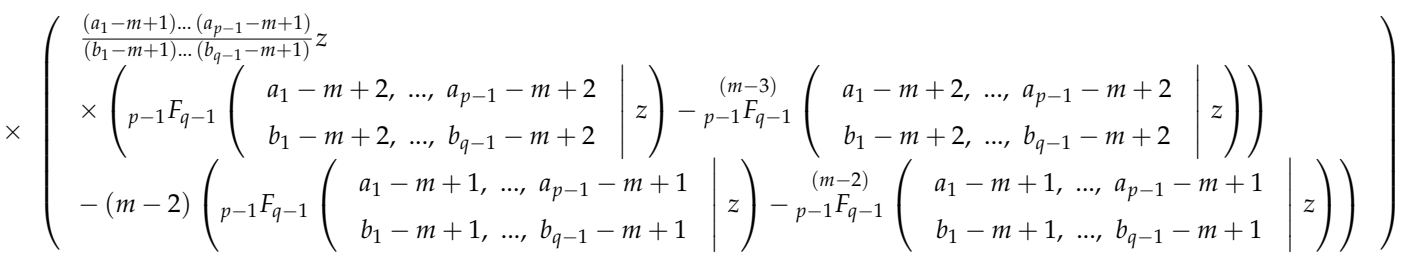

For instance, if $m=3$, Relation (30) reads as:

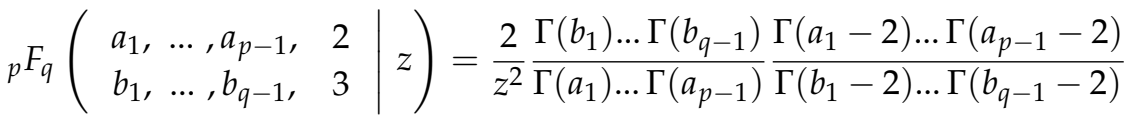

$$
\begin{aligned}
& \times\left(\frac{\left(a_{1}-2\right) \ldots\left(a_{p-1}-2\right)}{\left(b_{1}-2\right) \ldots\left(b_{q-1}-2\right)} z_{p-1} F_{q-1}\left(\begin{array}{ccc}
a_{1}-1, & \ldots, & a_{p-1}-1 \\
b_{1}-1, & \ldots, & b_{q-1}-1
\end{array} \mid z\right)\right. \\
& \left.-{ }_{p-1} F_{q-1}\left(\begin{array}{ccc}
a_{1}-2, & \ldots, & a_{p-1}-2 \\
b_{1}-2, & \ldots, & b_{q-1}-2
\end{array} \mid z\right)+1\right)
\end{aligned}
$$

Hence, for $p=q+1=3$ and $z=1$, we have:

$$
\begin{aligned}
{ }_{3} F_{2}\left(\begin{array}{ccc}
a, b, 2 & 1 \\
c, 3 & 1
\end{array}\right)=\frac{2}{(a-2)_{2}(b-2)_{2}} & \\
& \times\left((c-2)_{2}+\frac{\Gamma(c) \Gamma(c-a-b+1)}{\Gamma(c-a) \Gamma(c-b)}(a b-a-b-c+3)\right) .
\end{aligned}
$$

\section{Second Hypergeometric Identity}

By noting Relation (1), first it is not difficult to verify that:

$$
(a+m)_{k}=\frac{(a)_{k+m}}{(a)_{m}}
$$

Now, if the identity (31) is applied in a special case of (2), we obtain: 


$$
\begin{aligned}
{ }_{p} F_{q}\left(\begin{array}{cc}
a_{1}+m, \ldots a_{p-1}+m, & 1 \\
b_{1}+m, \ldots & , b_{q}+m
\end{array} \mid z\right)=\sum_{k=0}^{\infty} \frac{\left(a_{1}+m\right)_{k} \ldots\left(a_{p-1}+m\right)_{k}}{\left(b_{1}+m\right)_{k} \ldots\left(b_{q}+m\right)_{k}} z^{k} \\
=\frac{\left(b_{1}\right)_{m} \ldots\left(b_{q}\right)_{m}}{\left(a_{1}\right)_{m} \ldots\left(a_{p-1}\right)_{m}} \sum_{k=0}^{\infty} \frac{\left(a_{1}\right)_{k+m} \ldots\left(a_{p-1}\right)_{k+m}}{\left(b_{1}\right)_{k+m} \ldots\left(b_{q-1}\right)_{k+m}} z^{k} \\
=\frac{\left(b_{1}\right)_{m} \ldots\left(b_{q}\right)_{m}}{\left(a_{1}\right)_{m} \ldots\left(a_{p-1}\right)_{m}} \sum_{j=m}^{\infty} \frac{\left(a_{1}\right)_{j} \ldots\left(a_{p-1}\right)_{j}}{\left(b_{1}\right)_{j} \ldots\left(b_{q-1}\right)_{j}} z^{j-m} \\
=\frac{\left(b_{1}\right)_{m} \ldots\left(b_{q}\right)_{m}}{\left(a_{1}\right)_{m} \ldots\left(a_{p-1}\right)_{m}} z^{-m}\left(\sum_{j=0}^{\infty} \frac{\left(a_{1}\right)_{j} \ldots\left(a_{p-1}\right)_{j}}{\left(b_{1}\right)_{j} \ldots\left(b_{q-1}\right)_{j}} z^{j}-\sum_{j=0}^{m-1} \frac{\left(a_{1}\right)_{j} \ldots\left(a_{p-1}\right)_{j}}{\left(b_{1}\right)_{j} \ldots\left(b_{q-1}\right)_{j}} z^{j}\right),
\end{aligned}
$$

leading to the second identity:

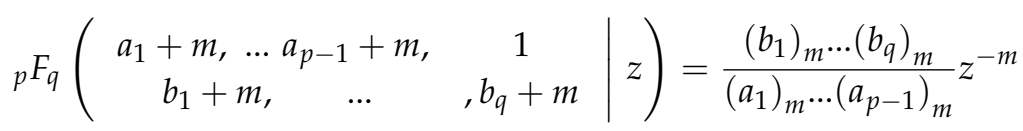

$$
\begin{aligned}
& \times\left({ }_{p} F_{q}\left(\begin{array}{ccc}
a_{1}, \ldots & a_{p-1}, & 1 \\
b_{1}, & \ldots
\end{array} \mid z\right)-{ }_{p}{ }_{p} F_{q}\left(\begin{array}{ccc}
a_{1}, \ldots & a_{p-1}, & 1 \\
b_{1}, & \ldots & , b_{q}
\end{array} \mid z\right)\right),
\end{aligned}
$$

which is equivalent to:

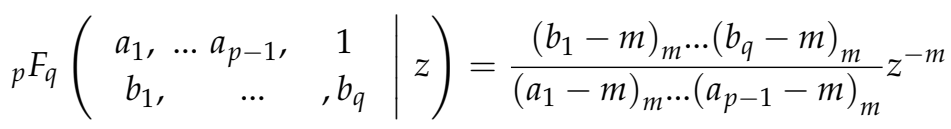

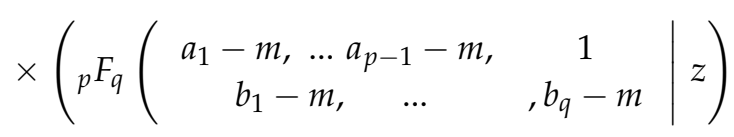

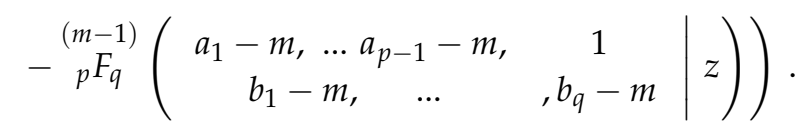

Once again, the interesting point is that by using Relation (32) or (33), various special cases can be considered as follows.

Special Case 12. When $p=2, q=1$ and $x=-1$, by noting the Kummer formula and Relation (32), we get:

$$
\begin{aligned}
{ }_{2} F_{1}\left(\begin{array}{c}
b+m, 1 \\
2-b+m
\end{array} \mid-1\right) & \\
= & (-1)^{m} \frac{(2-b)_{m}}{(b)_{m}}\left(\frac{\sqrt{\pi}}{2} \frac{\Gamma(2-b)}{\Gamma(-b+(3 / 2))}-{ }_{2}^{(m-1)} F_{1}\left(\begin{array}{c}
b, 1 \\
2-b
\end{array} \mid-1\right)\right) .
\end{aligned}
$$


For instance, if $m=1,2$, Relation (34) is simplified as:

$$
{ }_{2} F_{1}\left(\begin{array}{c}
b+1,1 \\
3-b
\end{array} \mid-1\right)=-\frac{\sqrt{\pi}}{2} \frac{\Gamma(3-b)}{\Gamma((3 / 2)-b)}+\frac{2}{b}-1,
$$

and:

$$
{ }_{2} F_{1}\left(\begin{array}{c|c}
b+2,1 & -1 \\
4-b & -1
\end{array}\right)=\frac{\sqrt{\pi}}{2 b(b+1)} \frac{\Gamma(4-b)}{\Gamma((3 / 2)-b)}-\frac{2(b-1)(b-3)}{b(b+1)}
$$

Special Case 13. When $p=2, q=1$ and $x=1 / 2$, by noting the second kind of Gauss formula and Relation (32), we get:

$$
\begin{aligned}
{ }_{2} F_{1}\left(\begin{array}{c|c}
a+m, 1 \\
(a / 2)+m+1
\end{array}\right. & \left.\frac{1}{2}\right) \\
& =\frac{(1+(a / 2))_{m}}{(a)_{m}} 2^{m}\left(\frac{\sqrt{\pi} \Gamma(1+(a / 2))}{\Gamma((a+1) / 2)}-{ }_{2}^{(m-1)}\left(\begin{array}{c}
a, 1 \\
(a / 2)+1
\end{array}\right)\right) .
\end{aligned}
$$

For instance, if $m=1,2$, Relation (35) is simplified as:

$$
{ }_{2} F_{1}\left(\begin{array}{c|c}
a+1,1 & 1 \\
(a / 2)+2 & 2
\end{array}\right)=\frac{a+2}{a}\left(\sqrt{\pi} \frac{\Gamma(1+(a / 2))}{\Gamma((a+1) / 2)}-1\right),
$$

and:

$$
{ }_{2} F_{1}\left(\begin{array}{c|c}
a+2,1 & 1 \\
(a / 2)+3 & \frac{2}{2}
\end{array}\right)=\sqrt{\pi} \frac{(a+2)(a+4)}{a(a+1)} \frac{\Gamma(1+(a / 2))}{\Gamma((a+1) / 2)}-2\left(1+\frac{4}{a}\right) .
$$

Special Case 14. When $p=3, q=2$ and $x=1$, by noting the Dixon formula and Relation (32), we get:

$$
\begin{aligned}
{ }_{3} F_{2}\left(\begin{array}{c|c}
b & +m, c+m, 1 \\
2- & b+m, 2-c+m
\end{array}\right)=\frac{(2-b)_{m}(2-c)_{m}}{(b)_{m}(c)_{m}} \\
\quad \times\left(\frac{\sqrt{\pi}}{2} \frac{\Gamma(2-b) \Gamma(2-c) \Gamma(-b-c+(3 / 2))}{\Gamma(-b+(3 / 2)) \Gamma(-c+(3 / 2)) \Gamma(2-b-c)}-{ }_{3}^{(m-1)} F_{2}\left(\begin{array}{c}
b, c, 1 \\
2-b, 2-c
\end{array}\right)\right)
\end{aligned}
$$

For instance, if $m=1,2$, Relation (36) is simplified as:

$$
\begin{array}{r}
{ }_{3} F_{2}\left(\begin{array}{c}
b+1, c+1,1 \\
3-b, 3-c
\end{array} \mid 1\right) \\
\quad=\frac{\sqrt{\pi}}{2 b c} \frac{\Gamma(3-b) \Gamma(3-c) \Gamma((3 / 2)-b-c)}{\Gamma((3 / 2)-b) \Gamma((3 / 2)-c) \Gamma(2-b-c)}-\frac{(b-2)(c-2)}{b c}
\end{array}
$$

and:

$$
\begin{gathered}
{ }_{3} F_{2}\left(\begin{array}{c|c}
b+2, c+2,1 & 1 \\
4-b, 4-c & 4
\end{array}\right) \\
\quad=\frac{\sqrt{\pi}}{2(b)_{2}(c)_{2}} \frac{\Gamma(4-b) \Gamma(4-c) \Gamma((3 / 2)-b-c)}{\Gamma((3 / 2)-b) \Gamma((3 / 2)-c) \Gamma(2-b-c)}-\frac{2(b c-b-c-2)(b-3)(c-3)}{(b)_{2}(c)_{2}}
\end{gathered}
$$


Special Case 15. When $p=3, q=2$ and $x=1$, by noting the Watson formula and Relation (32), we get:

$$
\begin{aligned}
& { }_{3} F_{2}\left(\begin{array}{c}
b+m, c+m, 1 \\
(b / 2)+m+1,2 c+m
\end{array} \mid 1\right)=\frac{(1+(b / 2))_{m}(2 c)_{m}}{(b)_{m}(c)_{m}} \\
& \quad \times\left(\frac{\sqrt{\pi} \Gamma(1+(c / 2)) \Gamma(1+(b / 2)) \Gamma(c-(b / 2))}{\Gamma((b+1) / 2) \Gamma(c) \Gamma(c-(b-1) / 2)}-{ }_{3}^{(m-1)} F_{2}\left(\begin{array}{c}
b, c, 1 \\
(b / 2)+1,2 c
\end{array}\right)\right) .
\end{aligned}
$$

For instance, if $m=1,2$, Relation (37) is simplified as:

$$
{ }_{3} F_{2}\left(\begin{array}{c|c}
b+1, c+1,1 \\
(b / 2)+2,2 c+1
\end{array} \mid 1\right)=\left(1+\frac{2}{b}\right)\left(\frac{\sqrt{\pi} \Gamma(1+(c / 2)) \Gamma(1+(b / 2)) \Gamma(c-(b / 2))}{\Gamma((b+1) / 2) \Gamma(c) \Gamma(c-(b-1) / 2)}-1\right)
$$

and:

$$
\begin{aligned}
{ }_{3} F_{2}\left(\begin{array}{cc|c}
b+2, c+2, & 1 & 1 \\
(b / 2)+3,2 c & +2 & 1
\end{array}\right) \\
\quad=\frac{2 \sqrt{\pi} c(2 c+1)}{b(b+1)} \frac{\Gamma((c / 2)+1) \Gamma((b / 2)+3) \Gamma(c-(b / 2))}{\Gamma((b+1) / 2) \Gamma(c+2) \Gamma(c-(b-1) / 2)}-\frac{(b+4)(2 c+1)}{b(c+1)} .
\end{aligned}
$$

Special Case 16. When $p=3, q=2$ and $x=1$, by noting the Whipple formula and Relation (32), we get:

$$
\begin{aligned}
& { }_{3} F_{2}\left(\begin{array}{c|c}
a+m, 1-a+m, 1 \\
c+m & 3-c+m
\end{array}\right)=\frac{(c)_{m}(3-c)_{m}}{(a)_{m}(1-a)_{m}} \\
& \times\left(\frac{\pi \Gamma(c) \Gamma(3-c)}{2 \Gamma((a+c) / 2) \Gamma((a-c+3) / 2) \Gamma((1-a+c) / 2) \Gamma(2-(a+c) / 2)}\right. \\
& \left.\quad-{ }_{3}^{(m-1)}\left(\begin{array}{cc|c}
a, 1-a, 1 & 1 \\
c, 3-c & 1
\end{array}\right)\right) .
\end{aligned}
$$

For instance, if $m=1,2$, Relation (38) is simplified as:

$$
\begin{aligned}
& { }_{3} F_{2}\left(\begin{array}{c|c}
a+1,2-a, 1 & 1 \\
c & +1,4-c
\end{array}\right) \\
& \quad=\frac{\pi}{2 a(1-a)} \frac{\Gamma(c+1) \Gamma(4-c)}{\Gamma((a+c) / 2) \Gamma((a-c+3) / 2) \Gamma((1-a+c) / 2) \Gamma(2-(a+c) / 2)}-\frac{c(c-3)}{a(a-1)},
\end{aligned}
$$

and:

$$
\begin{aligned}
{ }_{3} F_{2}\left(\begin{array}{c|c}
a+2,3-a, & 1 \\
c+2,5-c & 1
\end{array}\right) \\
\quad=\frac{\pi}{2(a-2)_{4}} \frac{\Gamma(c+2) \Gamma(5-c)}{\Gamma((a+c) / 2) \Gamma((a-c+3) / 2) \Gamma((1-a+c) / 2) \Gamma(2-(a+c) / 2)}
\end{aligned}
$$




$$
-\frac{(c+1)(4-c)(c(3-c)+a(1-a))}{(a-2)_{4}}
$$

Special Case 17. When $p=3, q=2$ and $x=1$, by noting the Pfaff-Saalschutz formula and Relation (32), we get:

$$
\begin{aligned}
& { }_{3} F_{2}\left(\begin{array}{c}
b+m,-n+m, 1 \\
c+m, 2+b-c-n+m
\end{array}\right. \\
& \quad=\frac{(c)_{m}(2+b-c-n)_{m}}{(b)_{m}(-n)_{m}}\left(\frac{(c-1)(c-1-b+n)}{(c-1-b)(c-1+n)}-{ }_{3}^{(m-1)} F_{2}\left(\begin{array}{c}
b,-n, 1 \\
c, 2+b-c-n
\end{array}\right)\right) .
\end{aligned}
$$

For instance, if $m=1,2$, Relation (39) is simplified as:

$$
{ }_{3} F_{2}\left(\begin{array}{c|c}
b+1,-n+1,1 \\
c+1,3+b-c-n
\end{array} \mid 1\right)=\frac{c(c-2-b+n)}{n b}\left(\frac{(c-1)(c-1-b+n)}{(c-1-b)(c-1+n)}-1\right),
$$

and:

$$
\begin{aligned}
{ }_{3} F_{2}\left(\begin{array}{cc}
b+2,-n+2, & 1 \\
c+2,4+b-c-n & 1
\end{array}\right) \\
=\frac{(c-1) c(c+1)(c-1-b+n)(c-2-b+n)(c-3-b+n)}{n(n-1) b(b+1)(c-1-b)(c-1+n)} \\
-\frac{(c+1)(c-3-b+n)(n b+c(c-2-b+n))}{n(n-1) b(b+1)} .
\end{aligned}
$$

Special Case 18. When $p=4, q=3$ and $x=-1$, by noting the Whipple formula and Relation (32), we get:

$$
\begin{aligned}
& { }_{4} F_{3}\left(\begin{array}{c}
m+(3 / 2), \quad b+m, c+m, 1 \\
m+(1 / 2), 2-b+m, 2-c+m
\end{array} \mid-1\right)=\frac{(-1)^{m}}{2 m+1} \frac{(2-b)_{m}(2-c)_{m}}{(b)_{m}(c)_{m}} \\
& \times\left(\frac{\Gamma(2-b) \Gamma(2-c)}{\Gamma(2-b-c)}-{ }_{4}^{(m-1)} F_{3}\left(\begin{array}{c}
3 / 2, b, c, 1 \\
1 / 2,2-b, 2-c
\end{array} \mid-1\right)\right) .
\end{aligned}
$$

For instance, if $m=1,2$, Relation (40) is simplified as:

$$
{ }_{4} F_{3}\left(\begin{array}{c}
5 / 2, b+1, c+1,1 \\
3 / 2,3-b, 3-c
\end{array} \mid-1\right)=\frac{1}{3 b c}\left((b-2)(c-2)-\frac{\Gamma(3-b) \Gamma(3-c)}{\Gamma(2-b-c)}\right)
$$

and:

$$
{ }_{4} F_{3}\left(\begin{array}{c}
7 / 2, b+2, c+2,1 \\
5 / 2,4-b, 4-c
\end{array} \mid-1\right)=\frac{(2-b)_{2}(2-c)_{2}}{5(b)_{2}(c)_{2}}
$$




$$
\times\left(\frac{\Gamma(2-b) \Gamma(2-c)}{\Gamma(2-b-c)}+2 \frac{b c+b+c-2}{(b-2)(c-2)}\right) .
$$

Special Case 19. When $p=5, q=4$ and $x=1$, by noting the Dougall formula and Relation (32), we get:

$$
\begin{aligned}
& { }_{5} F_{4}\left(\begin{array}{c|c}
m+(3 / 2), c+m, d+m, e+m, 1 \\
m+(1 / 2), 2-c+m, 2-d+m, 2-e+m
\end{array} \mid 1\right) \\
& =\frac{1}{2 m+1} \frac{(2-c)_{m}(2-d)_{m}(2-e)_{m}}{(c)_{m}(d)_{m}(e)_{m}} \\
& \times\left(\frac{\Gamma(2-c) \Gamma(2-d) \Gamma(2-e) \Gamma(2-c-d-e)}{\Gamma(2-d-e) \Gamma(2-c-e) \Gamma(2-c-d)}-{ }_{5}^{(m-1)} F_{4}\left(\begin{array}{c}
3 / 2, c, d, e, 1 \\
1 / 2,2-c, 2-d, 2-e
\end{array}\right)\right) .
\end{aligned}
$$

For instance, if $m=1,2$, Relation (41) is simplified as:

$$
\begin{aligned}
{ }_{5} F_{4}\left(\begin{array}{c|c}
5 / 2, c+1, d+1, e+1,1 \\
3 / 2,3-c, 3-d, 3-e
\end{array} \mid 1\right)= & \frac{(2-c)(2-d)(2-e)}{3 c d e} \\
& \times\left(\frac{\Gamma(2-c) \Gamma(2-d) \Gamma(2-e) \Gamma(2-c-d-e)}{\Gamma(2-d-e) \Gamma(2-c-e) \Gamma(2-c-d)}-1\right),
\end{aligned}
$$

and:

$$
\begin{aligned}
& { }_{5} F_{4}\left(\begin{array}{c|c}
7 / 2, c+2, d+2, e+2,1 & 1 \\
5 / 2,4-c, 4-d, 4-e & 1
\end{array}\right)=\frac{(2-c)_{2}(2-d)_{2}(2-e)_{2}}{5(c)_{2}(d)_{2}(e)_{2}} \\
& \times\left(\frac{\Gamma(2-c) \Gamma(2-d) \Gamma(2-e) \Gamma(2-c-d-e)}{\Gamma(2-d-e) \Gamma(2-c-e) \Gamma(2-c-d)}-\frac{3 c d e}{(2-c)(2-d)(2-e)}-1\right) .
\end{aligned}
$$

Special Case 20. When $p=7, q=6$ and $x=1$, by noting the Dougall formula and Relation (32), we get:

$$
\begin{aligned}
& { }_{7} F_{6}\left(\begin{array}{c|c}
m+(3 / 2), b+m, c+m, d+m, 3-b-c+n+m, m-n, 1 \\
m+(1 / 2), 2-b+m, 2-c+m, 2-d+m, b+c+d-n-1+m, n+2+m & 1
\end{array}\right) \\
& =\frac{1}{2 m+1} \frac{(2-b)_{m}(2-c)_{m}(2-d)_{m}(b+c+d-n-1)_{m}(n+2)_{m}}{(b)_{m}(c)_{m}(d)_{m}(3-b-c+n)_{m}(-n)_{m}} \\
& \times\left(\frac{(2)_{n}(2-b-c)_{n}(2-b-d)_{n}(2-c-d)_{n}}{(2-b)_{n}(2-c)_{n}(2-d)_{n}(2-b-c-d)_{n}}\right. \\
& \left.-{ }_{7} F_{6}\left(\begin{array}{c|c}
3 / 2, b, c, d, 3-b-c+n,-n, 1 \\
1 / 2,2-b, 2-c, 2-d, b+c+d-n-1, n+2
\end{array}\right)\right) .
\end{aligned}
$$


For instance, if $m=1,2$, Relation (42) is simplified as:

$$
\begin{array}{r}
{ }_{7} F_{6}\left(\begin{array}{c|c}
5 / 2, b+1, c+1, & d+1,3-b-c+n+1,1-n, 1 \\
3 / 2,3-b, 3-c, 3-d, b+c+d-n, n+3 & 1
\end{array}\right) \\
=\frac{(2-b)(2-c)(2-d)(b+c+d-n-1)(n+2)}{3 n b c d(3-b-c+n)} \\
\times\left(1-\frac{(2)_{n}(2-b-c)_{n}(2-b-d)_{n}(2-c-d)_{n}}{(2-b)_{n}(2-c)_{n}(2-d)_{n}(2-b-c-d)_{n}}\right)
\end{array}
$$

and:

$$
\begin{gathered}
{ }_{7} F_{6}\left(\begin{array}{c}
7 / 2, b+2, c+2, d+2,5-b-c+n, 2-n, 1 \\
5 / 2,4-b, 4-c, 4-d, b+c+d-n+1, n+4
\end{array}\right) \\
=\frac{(2-b)_{2}(2-c)_{2}(2-d)_{2}(b+c+d-n-1)_{2}(n+2)_{2}}{5(b)_{2}(c)_{2}(d)_{2}(3-b-c+n)_{2}(-n)_{2}} \\
\times\left(\frac{(2)_{n}(2-b-c)_{n}(2-b-d)_{n}(2-c-d)_{n}}{(2-b)_{n}(2-c)_{n}(2-d)_{n}(2-b-c-d)_{n}}\right. \\
\left.+\frac{3 b c d n(3-b-c+n)}{(2-b)(2-c)(2-d)(b+c+d-n-1)(n+2)}-1\right) .
\end{gathered}
$$

\section{Conclusions}

In this paper, we applied two identities for generalized hypergeometric series in order to extend some classical summation theorems of hypergeometric functions such as Gauss, Kummer, Dixon, Watson, Whipple, Pfaff-Saalschütz and Dougall formulas and then obtained some new summation theorems using the second introduced hypergeometric identity.

Author Contributions: Both authors have the same contribution in all sections.

Acknowledgments: This research was funded by the Alexander von Humboldt Foundation under Grant Number Ref 3.4-IRN-1128637-GF-E.

Conflicts of Interest: The authors declare no conflict of interest.

\section{References}

1. Koepf, W. Hypergeometric Summation: An Algorithmic Approach to Summation and Special Function Identities, 2nd ed.; Springer: London, UK, 2014.

2. Slater, L.J. Generalized Hypergeometric Functions; Cambridge University Press: Cambridge, UK, 1966.

3. Andrews, G.E.; Askey, R.; Roy, R. Special Functions. In Encyclopedia of Mathematics and Its Applications; Cambridge University Press: Cambridge, UK, 1999; Volume 71.

4. Arfken, G. Mathematical Methods for Physicists; Academic Press: New York, NY, USA, 1985.

5. Mathai, A.M.; Saxena, R.K. Generalized Hypergeometric Functions with Applications in Statistics and Physical Sciences; Lecture Notes in Mathematics; Springer: Berlin/Heidelberg, Germany; New York, NY, USA, 1973; Volume 348.

6. Nikiforov, A.F.; Uvarov, V.B. Special Functions of Mathematical Physics. A Unified Introduction with Applications; Birkhauser: Basel, Switzerland, 1988. 
7. Bailey, W.N. Products of generalized hypergeometric series. Proc. Lond. Math. Soc. 1928, 2, 242-254. [CrossRef]

8. Karlsson, P.W. Hypergeometric functions with integral parameter differences. J. Math. Phys. 1971, 12, $270-271$. [CrossRef]

9. Miller, A.R. Certain summation and transformation formulas for generalized hypergeometric series. J. Comput. Appl. Math. 2009, 231, 964-972. [CrossRef]

10. Miller, A.R.; Srivastava, H.M. Karlsson-Minton summation theorems for the generalized hypergeometric series of unit argument. Integral Transform. Spec. Funct. 2010, 21, 603-612. [CrossRef]

11. Minton, B.M. Generalized hypergeometric function of unit argument. J. Math. Phys. 1970, 11, 1375-1376. [CrossRef]

12. Rosengren, H. Karlsson-Minton type hypergeometric functions on the root system $C_{n}$. J. Math. Anal. Appl. 2003, 281, 332-345. [CrossRef]

13. Srivastava, H.M. Generalized hypergeometric functions with integral parameter differences. Indag. Math. 1973, 76, 38-40. [CrossRef]

14. Prudnikov, A.P.; Brychkov, Y.A.; Marichev, O.I. More Special Functions; Integrals and Series; Gordon and Breach Science Publishers: Amsterdam, The Netherlands, 1990; Volume 3.

(C) 2018 by the authors. Licensee MDPI, Basel, Switzerland. This article is an open access article distributed under the terms and conditions of the Creative Commons Attribution (CC BY) license (http:/ / creativecommons.org/licenses/by/4.0/). 